\title{
A Framework for Estimation of Power Systems Based on Synchronized Phasor Measurement Data
}

\author{
Luigi Vanfretti, Student Member, IEEE, Joe H. Chow, Fellow, IEEE, \\ Sanjoy Sarawgi, Member, IEEE, Dean Ellis and Bruce Fardanesh, Senior Member, IEEE
}

\begin{abstract}
This paper presents a new approach for synchronized phasor measurement-based state estimation, which starts from the buses with synchronized phasor data and enables highly accurate estimation of portions of the power system with connectivity to these phasor data buses. Observability analysis is used to assemble the buses for this Phasor State Estimator (PSE). The state estimation is formulated as an iterative leastsquares problem, with bus voltage and line current magnitudes and phases as the estimated variables. An important aspect of this formulation is its ability to correct for constant or random phase biases that may exist in some phasor measurement equipment. Beside being a standalone state estimator, the PSE can enhance the reliability of a conventional state estimator. The PSE methodology is demonstrated for a power transfer path in the New York power system.
\end{abstract}

Index Terms-synchronized phasor measurements, phasor state estimation, observability methodologies, measurement placement.

\section{INTRODUCTION}

A S conceived during the 1970's [1], [2], [3], [4] State Estimation (SE) provides static estimates of the system states using measurements from units such as RTUs [2]. Currently SEs use the Intercontrol Center Communications Protocol (ICCP) gathering asynchronously fed data with an arrival rate of 1 sample per 4-10 seconds. Even though there have been great efforts to improve the the detection of bad data and topology errors [2], [3], [5], there is still a need to provide better bad data detection and more accurate models for the external systems. With the advancement of synchrophasor technology there has been interests to use Phasor Measurement Unit (PMU) measurements to enhance the estimates provided by SEs used in control centers [6]. In the case when a PMU is applied to every bus on the system, [7] establishes a noniterative least-squares solution to perform state estimation. Assuming far fewer PMUs, [8] suggests to install PMUs at critical buses to enhance the reliability of conventional SEs. ${ }^{1}$

In this paper, we investigate a phasor state estimator (PSE) implementation in a power system where a number of PMUs have been installed on high-voltage (HV) substations, although not necessarily on every HV substation. This new PSE will be

L. Vanfretti and J. H. Chow are with the Department of Electrical, Computer, and Systems Engineering, Rensselaer Polytechnic Institute, Troy, NY 12180, USA. (e-mail: vanfrl@rpi.edu, chowj@rpi.edu).

S. Sarawgi is with American Electric Power, Gahanna, OH 43230, USA. (e-mail: sksarawgi@aep.com)

D. Ellis is with NYISO, Rensselaer, NY 12144, USA. (e-mail: dellis@nyiso.com)

B. Fardanesh is with NYPA and Manhattan College, NY. (e-mail: Bruce.Fardanesh@nypa.gov)

${ }^{1}$ Loss of ICCP data at critical buses will result in unobservable islands. built on synchronized phasor data only, and its solutions can be used to supplement a conventional SE based on ICCP data. Such a schematic is shown in Fig. 1. This approach is attractive because the PSE is built independent of the conventional SE, allowing the PSE to be implemented without disrupting the role of a conventional SE in control centers.

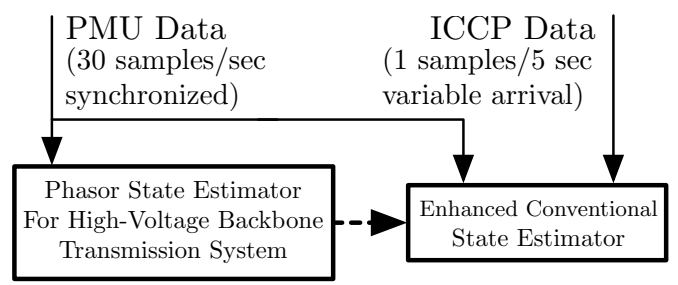

Figure 1. Hybrid Sate Estimation

The formulation of the proposed PSE allows us to perform a specific type of phasor angle correction that does not exist in conventional SE. We have observed that in some PMUs, the voltage and current phasor angles can exhibit a constant bias due to the signal processing techniques used to determine the phasors. Some other PMUs exhibit random angle jumps, typically multiples of $7.5^{\circ}$, due to unknown reasons [9]. However, the fact that the same angle shift will show up in all measurement channels in a PMU allows the PSE to correct the angle shifts. This is important because such uncorrected phasor data will cause convergence difficulties if used in conventional SEs.

The remainder of this paper is organized as follows. Section II develops the PSE network model and Section III introduces the state estimation solution algorithm. Angle bias correction is discussed in Section IV, which includes an observability analysis. The application of the proposed PSE to actual PMU data recorded in the New York power system is demonstrated in Section V.

\section{PSE Network Model}

We first determine the buses and lines in the PSE network model, and the corresponding equations to calculate the voltages and flows in the PSE model. In the sequel, if a PMU is installed on a bus, we will refer to it as a PMU bus. Otherwise, it is a non-PMU bus.

\section{A. Model Buses and Lines}

Consider a power system with $N$ buses. We label these buses as $i=1,2, \ldots, N$, and their corresponding bus voltage phasors as $\tilde{V}_{i}=V_{i} \varepsilon^{j \theta_{i}}, i=1,2, \ldots, N_{1}$. The measurements 
for these voltage phasors are denoted as $\tilde{V}_{i m}=V_{i m} \varepsilon^{j \theta_{i m}}$, $i=1,2, \ldots, N_{1}$.

The PMUs also measure a number $L_{1}$ of line current flows between the buses. The current going from a PMU Bus $i$ to another Bus $k$, whether a PMU bus or a non-PMU bus, will be denoted by $\tilde{I}_{i k}=I_{i k} \varepsilon^{j \delta_{i k}}$, whose measurements will be denoted as $\tilde{I}_{i k m}=I_{i k m} \varepsilon^{j \delta_{i k m}}$. Let the total number of nonPMU buses be denoted by $N_{2}$, and without loss of generality, these buses will be labeled as $i=N_{1}+1, N_{1}+2, \ldots, N_{1}+$ $N_{2}$. In addition, let the total number of lines without phasor measurement connecting the PMU and non-PMU buses be $L_{2}$.

Thus the collection of these $N=N_{1}+N_{2}$ buses and $L=L_{1}+L_{2}$ branches connecting these buses comprises the PSE network model. Note that in this PSE model, a branch connecting two PMU buses, whose current flow is measured on both ends, will be counted twice. This would not cause any problem in the subsequent state estimation process.

An example of such a PSE network is shown in Fig. 2. The voltage phasors are measured at the PMU Buses 1, 2, and 3. These PMUs also measure the current phasors on Lines 1-4, 2-5, 3-6, and 3-7. Thus the non-PMU Buses 4, 5, 6, and 7, and Lines 1-7, 2-4, 2-6, 4-5, 5-6, and 5-7 are also included in the PSE network, which has a total of 7 buses and 10 lines. Note that connections from these 7 buses to other buses are not shown in Fig. 2.

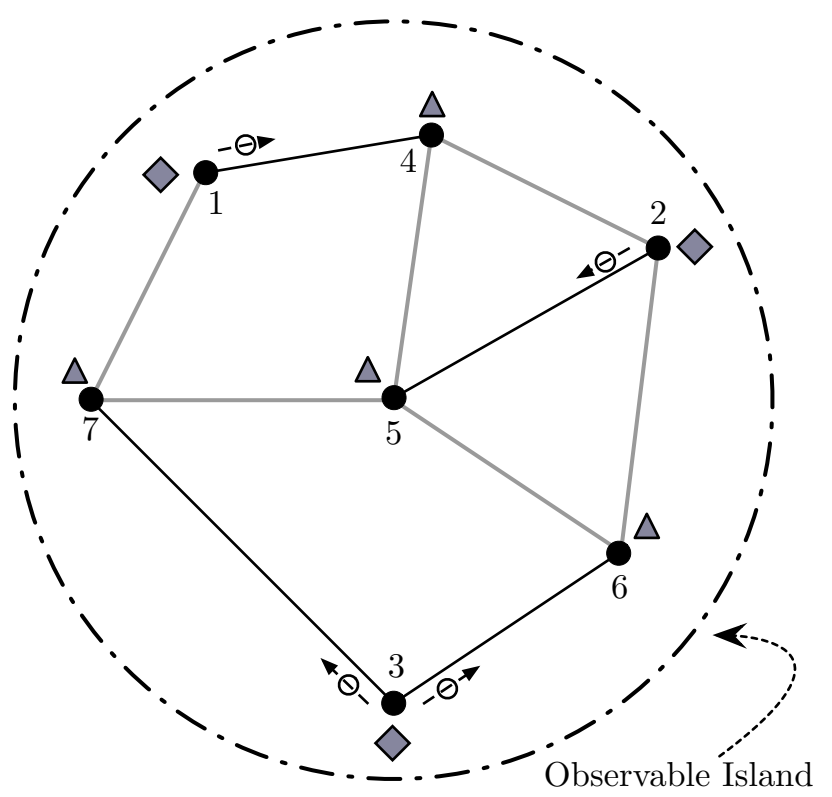

PMU Node $-\Theta \rightarrow$ PMU Current $\triangle$ Estimated Node

Figure 2. PSE Observable Islands (Network 1)

\section{B. Observability Analysis}

From the PSE network model, we can proceed to determine whether there are observable islands within the model. If every bus in the PSE model is connected to all the other buses via the branches available in the model, there the PSE is a single island. Otherwise, one can use an iterative algorithm to isolate the islands, such as those available in [2], [3]. In the sequel, we will assume that the PSE model consists of a single island, such that all the voltages of the $N$ buses and the currents on the $L$ branches connecting them will be observable.

\section{Network Model}

The network model is developed based on circuit equations, using the equivalent circuit in Fig. 3 connecting any two buses in the PSE network. The branch connecting Buses $i$ and $k$ is represented by the transmission line with impedance $\tilde{Z}_{i k}=$ $R_{i k}+j X_{i k}$ and line charging $\tilde{Y}_{i k}=j B_{i k}$. The current phasor leaving Bus i as $\tilde{I}_{i k}$ is related to the bus voltages and line parameters as

$$
\tilde{V}_{k}=\tilde{V}_{i}-Z_{i k}\left(\tilde{I}_{i k}-\frac{1}{2} Y_{i k} \tilde{V}_{i}\right)
$$

Note that there will be $L$ such complex equations for the PSE network. Each circuit equation in complex form can be decomposed into 2 equations, one for the real part and the other for the imaginary part, respectively, as

$$
f_{i k \mathrm{re}}=\operatorname{Re}\left(f_{i k}\right)=0, \quad f_{i k \mathrm{im}}=\operatorname{Im}\left(f_{i k}\right)=0
$$

where

$$
f_{i k}=\left(1+\frac{1}{2} Y_{i k} Z_{i k}\right) \tilde{V}_{i}-Z_{i k} \tilde{I}_{i k}-\tilde{V}_{k}
$$

We define the $2 L$ dimensional vector $f$

$$
f=\left[\begin{array}{llll}
\cdots & f_{i k \mathrm{re}} & f_{i k \mathrm{im}} & \cdots
\end{array}\right]^{T}
$$

of all the lines. Furthermore, the branches with current phasor measurements will occupy the first $2 L_{1}$ rows of $f$.

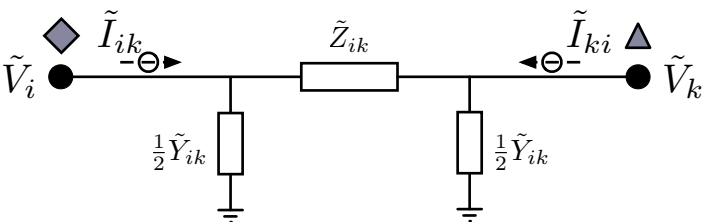

PMU Node $-\Theta>$ PMU Current $\triangle$ Estimated Node Network Node

Figure 3. PMU Bus- $i$ Circuit for the PSE Network Model

\section{Measurement Model}

At each PMU Bus the available measurements are $V_{i m}$, $\theta_{i m}, I_{i k m}$, and $\delta_{i k m}$. The measurement equations are divided into voltage measurement equations and current measurement equations.

1) Voltage Measurement Equations: are constructed by using the $V_{i m}$ and $\theta_{i m}$ measurements at each PMU-bus and equating them to their corresponding state resulting in

$$
V_{i}=V_{i m}+e_{V_{i}}, \quad \theta_{i}=\theta_{i m}+e_{\theta_{i}}
$$

where $e_{V_{i}}$ and $e_{\theta_{i}}$ are measurement errors. 
2) Current Measurement Equations: are constructed by using the $I_{i k m}$ and $\delta_{i k m}$ measurements at each PMU bus for Line $i-k$ as

$$
I_{i k}=I_{i_{k} m}+e_{I_{i k}}, \quad \delta_{i k}=\delta_{i k m}+e_{\delta_{i k}}
$$

where $e_{I_{i k}}$ and $e_{\delta_{i k}}$ are measurement errors.

We point out that this magnitude and phase measurement error representation is more appropriate than a rectangular coordinate representation because the magnitude and phase are mostly uncorrelated variables as obtained by PMUs.

To summarize, the total number of unknown variables in the PSE model $2 N$ voltage magnitudes and angles, and $2 L$ line current magnitudes and angles. These unknown variables are arranged as a vector

$$
x=\left[\begin{array}{l}
V \\
I \\
\theta \\
\delta
\end{array}\right]
$$

where the voltage magnitude and angle vectors are respectively defined as

$$
\begin{aligned}
V & =\left[\begin{array}{llllll}
V_{1} & \cdots & V_{N_{1}} & V_{N_{1}+1} & \cdots & V_{N}
\end{array}\right]^{T} \\
\theta & =\left[\begin{array}{llllll}
\theta_{1} & \cdots & \theta_{N_{1}} & \theta_{N_{1}+1} & \cdots & \theta_{N}
\end{array}\right]^{T}
\end{aligned}
$$

and the current magnitude and angle vectors are respectively defined as

$$
\begin{aligned}
I & =\left[\begin{array}{lll}
\cdots & I_{i k} & \cdots
\end{array}\right]^{T} \\
\delta & =\left[\begin{array}{lll}
\cdots & \delta_{i k} & \cdots
\end{array}\right]^{T}
\end{aligned}
$$

for all the lines where the measured current magnitudes and angles are placed in the first parts of these vectors. These $U_{T}=2(N+L)$ unknown variables are constrained by $E_{T}=$ $2\left(L+N_{1}+L_{1}\right)$ real equations arising from (1), (5), and (6) of the PSE model. Note that from the construction of the PSE network described in Section II, $L_{1} \geq N_{2}$. Strict inequality occurs when, for example, a non-PMU bus is connected to more than one PMU bus and the current phasors on all the connecting branches are measured. Thus

$$
L+N \leq L+N_{1}+L_{1}
$$

and the PSE network is observable. As an example, for the PSE network in Fig. 2, $L_{1}=N_{2}=4$. Thus (12) holds as an equality.

\section{Phasor State Estimation}

\section{A. Least-Squares Formulation}

The objective of the PSE problem is to find a set of voltage and current phasors satisfying (1) while minimizing the measurement errors in the measurement equations (5) and (6). In the case $L_{1}=N_{2}$, the number of unknown variables is equal to the number of constrained equations. Thus a unique solution for the unknown variables exists, where the measurement errors are taken to be zero. On the other hand, if $L_{1}>N_{2}$, then the PSE can be formulated as a nonlinear weighted least-squares (WLS) problem [10], [11]. Define the objective function as

$$
\begin{aligned}
q(x)=\frac{1}{2}( & \left\|W_{V} e_{V}\right\|^{2}+\left\|W_{\theta} e_{\theta}\right\|^{2} \\
+ & \left.\left\|W_{I} e_{I}\right\|^{2}+\left\|W_{\delta} e_{\delta}\right\|^{2}\right)
\end{aligned}
$$

where $\|\cdot\|$ denotes the magnitude of its vector argument, $e_{V}$, $e_{\theta}, e_{I}$, and $e_{\delta}$ are vectors of the measurement errors in (5) and (6). The weighting matrices in (13) are diagonal matrices given by

$$
\begin{aligned}
& W_{V}=\operatorname{diag}\left(\cdots, \min \left(1, \frac{1}{V_{i m}}\right), \cdots\right) \\
& W_{\theta}=\operatorname{diag}\left(\cdots, \min \left(1, \frac{1}{\left|\theta_{i m}\right|}\right), \cdots\right) \\
& W_{I}=\operatorname{diag}\left(\cdots, \min \left(1, \frac{1}{I_{i k m}}\right), \cdots\right) \\
& W_{\delta}=\operatorname{diag}\left(\cdots, \min \left(1, \frac{1}{\left|\delta_{i k m}\right|}\right), \cdots\right)
\end{aligned}
$$

Most of the weights will be about unity, except for weights on the current magnitude which can be lower for heavily loaded lines.

The WLS problem can be formulated as

$$
\min _{x} q(x), \text { subject to : } f=0
$$

Because $f$ is a nonlinear function of $V, I, \theta$, and $\delta$, we augment the equality constraint $f=0$ to the objective function $q(x)$ to form

$$
q^{\prime}(x)=q(x)+\frac{1}{2}\left\|W_{f} f\right\|^{2}=\frac{1}{2}\|W h(x)\|^{2}
$$

where $W_{f}$ is diagonal matrix of large weights,

$$
\begin{aligned}
& h=\left[\begin{array}{ll}
f^{T} & e^{T}
\end{array}\right]^{T}, \quad e=\left[\begin{array}{llll}
e_{V}^{T} & e_{\theta}^{T} & e_{I}^{T} & e_{\delta}^{T}
\end{array}\right]^{T} \\
& W=\text { block }-\operatorname{diag}\left(\begin{array}{cllll}
W_{f} & W_{V} & W_{\theta} & W_{I} & W_{\delta}
\end{array}\right)
\end{aligned}
$$

Thus the constrained WLS problem (18) is transformed into an unconstrained WLS problem of

$$
\min _{x} q^{\prime}(x)
$$

\section{B. Successive Phasor State Estimation Algorithm}

There are a number of approaches to solve the nonlinear WLS problem (22). A Newton algorithm would the second derivative of $q^{\prime}$. Here we use the Gauss-Newton Method, which requires only the first derivative of $q^{\prime}$.

In the Gauss-Newton method, starting from the current value $x_{c}$ of the solution vector $x$, the increment in $x$ is computed as

$$
\Delta x=-\left(H\left(x_{c}\right)\right)^{-1}\left(W J\left(x_{c}\right)\right)^{T} h\left(x_{c}\right)
$$

where the Jacobian matrix is

$$
J(x)=\partial h(x) / \partial x
$$

and

$$
H=\left(W J\left(x_{c}\right)\right)^{T}\left(W J\left(x_{c}\right)\right)
$$

The new solution is updated to $x_{c}+\Delta x$ and the Gauss-Newton iteration (23) is repeated until the solution converges. Note that the convergence of the Gauss-Newton iteration is quadratic when the solution is close to the optimum value of $x$. 


\section{Jacobian Matrix Structure}

Following the arrangements of the unknown variables in $x$ (7) and the network equations (20), the structure of the Jacobian (24) has the following form

$J=\left[\begin{array}{c|c|c|c|c|c|c|c}\hline \multicolumn{7}{c}{\partial f / \partial x} \\ \hline 0 & 0 & 0 & 0 & 0 & 0 & 0 & 0 \\ \hline 0 & 0 & 0 & 0 & U & 0 & 0 & 0 \\ \hline 0 & 0 & U & 0 & 0 & 0 & 0 & 0 \\ \hline & 0 & 0 & 0 & 0 & U & 0\end{array}\right]$

where $U$ is the identity matrix with appropriate dimension, which arises from the partial derivatives of the measurement error vector with respect to its own variables. Note that the Jacobian is very sparse. Algorithms [10] exploiting sparsity can be used solve for the increment $\Delta x$ in (23) for computational efficiency.

\section{EXTENSION OF PSE FOR ANGLE BiAs CORRECTION}

Both persistent and random angle biases in phasor data have been observed in PMUs installed in utilities [9]. A WLS approach is suitable for detecting and correcting such angle biases. The ability for a PSE network to perform angle bias correction requires that there are redundant phasor measurements, that is, $L_{1}>N_{2}$. For example, consider the two PSE networks shown in Figure 4, each with the indicated voltage and current phasor measurements. In Fig. $4 \mathrm{a}, \tilde{V}_{3}$ can be computed using the phasor measurements from either Bus 1 or Bus 2. If one of the phasor measurements has an angle bias, the two computed values of $\tilde{V}_{3}$ will be different. If the angle bias is corrected, then all 5 voltages in the network can be accurately computed. A simpler example system in given in Fig. $4 \mathrm{~b}$ where the voltage phasor measurements at adjacent buses provide a check on the phasor current.

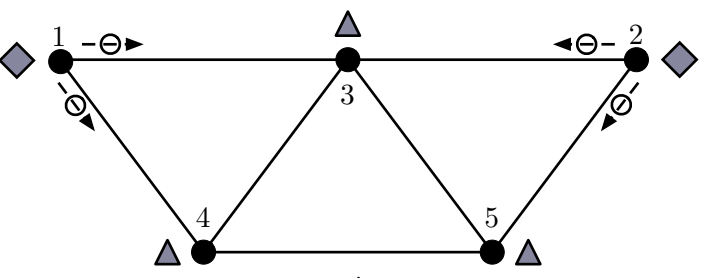

PMU Node $-\Theta>$ PMU Current $\triangle$ Estimated Node Network Node

(a) Five-Bus PSE Network (Network 2)

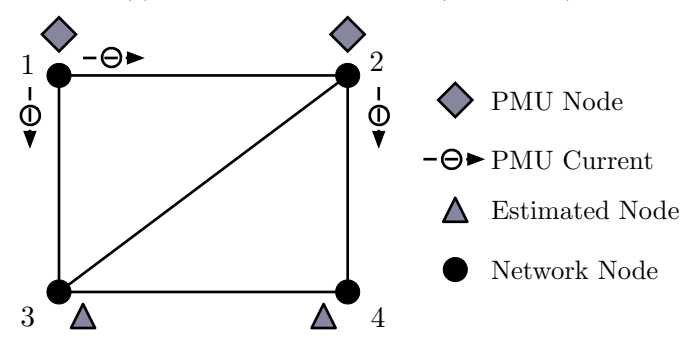

(b) Four-Bus PSE Network (Network 3)

Figure 4. Example networks allowing angle bias correction

\section{A. Modification of Measurement Equations}

There are several potential sources of angle biases. It could be due to a particular signal processing algorithm used by the PMU and the length of the data used for computing the phasor quantities. It could also be attributed to time delays due to long cables or errors in time synchronization. PMU software and firm ware may also contribute to the error.

On the other hand, this kind of angle bias has the characteristic that when large angle bias occurs, the same value appears in all voltage and current angles. Thus in the WLS algorithm formulation, the measurement equations (5) and (6) can be updated to, respectively,

$$
\theta_{i}=\theta_{i m}-\phi_{i}+e_{\theta_{i}}
$$

and

$$
\delta_{i k}=\delta_{i k m}-\phi_{i}+e_{\delta_{i k}}
$$

Note that we take Bus 1 to be the reference bus, and thus the angle bias will not be applied to its phasor measurements. The angle bias terms form a vector

$$
\phi=\left[\begin{array}{llll}
\phi_{2} & \phi_{3} & \cdots & \phi_{N_{1}}
\end{array}\right]^{T}
$$

Thus the WLS algorithm (22) can be modified to the PSE- $\Phi$ problem of

where

$$
\min _{x_{\phi}} q^{\prime}\left(x_{\phi}\right)
$$

$$
x_{\phi}=\left[\begin{array}{ll}
x^{T} & \phi^{T}
\end{array}\right]^{T}
$$

and $e\left(x_{\phi}\right)$ has been modified to incorporate (27) and (28).

Note that the Jacobian matrix is expanded to

$$
J_{\phi}=\left[\begin{array}{l|l}
J & 0 \\
\partial e(\phi) / \partial \phi
\end{array}\right]
$$

Note that the Jacobian $\partial e(\phi) / \partial \phi$ is sparse and consists of ones and zeros.

To correct for angle bias, it is necessary to have $L_{1}>N_{2}$. To ensure that all the biases can be corrected, the rank of $J_{\phi}$ must be equal to the number of unknown variables, which when including the angle biases, is $U_{T \phi}=2(N+L)+\left(N_{1}-1\right)$. In the sequel the term PSE- $\Phi$ is used to refer to a network where this conditions hold.

\section{B. PSE- $\Phi$ Observability Analysis for Test Networks}

Next we perform an observability analysis of Network 1 (Fig. 2), Network 2 (Fig. 4a), and Network 3 (Fig. 4b). For all three networks, the rank of the relevant Jacobian matrix $J$ is equal to the unknowns $U_{T}$, as shown in Table I, implying that they are all PSE observable. Now consider correcting potential biases on the PMU buses. For Network 1, $\phi_{2}$ and $\phi_{3}$ are added, resulting in a total of 36 unknown variables. The number of network equations, however, remains at 34 , thus implying the rank of $J_{\phi}$ is also 34 . Thus PMU angle biases cannot be corrected for Network 1. For Networks 2 and 3, when $\phi_{2}$ is included in the formulation, increasing the number of unknowns by 1 , the rank of $J_{\phi}$ is also one higher than the rank of $J$, ensuring that these two networks are PSE$\Phi$ observable. 
Table I

OBSERVABILITY ANALYSIS RESULTS

\begin{tabular}{|c|c|c|c|c|c|c|c|}
\hline NET. & $E_{T}$ & $U_{T}$ & $\operatorname{rank}(J)$ & Bias Terms & $U_{T \phi}$ & $\operatorname{rank}\left(J_{\phi}\right)$ & Observ. \\
\hline \hline 1 & 34 & 34 & 34 & $\phi_{2}, \phi_{3}$ & 36 & 34 & No \\
\hline 2 & 26 & 24 & 24 & $\phi_{2}$ & 25 & 25 & Yes \\
\hline 3 & 20 & 18 & 18 & $\phi_{2}$ & 19 & 19 & Yes \\
\hline
\end{tabular}

\section{Illustration with New York System}

Figure 5 shows the one-line diagram of $3 \mathrm{HV}$ buses in the New York power system. This 3-bus network comprises one transmission line and two transformers. PMUs are installed at Buses 1 and 2 providing voltage and current phasors at 6 samples per second. At Bus 1 the current flowing out from Bus 1 to Bus 3 is measured, while the Bus 2 currents flowing through the transformers are measured. With this measurement configuration this network is PSE and PSE- $\Phi$ observable.

Synchronized phasor measurements over a 4-minute period from this network are shown in Fig. 6 along with the solution from the Phasor State Estimation. The voltage and current phasors were corrupted by adding $7.5^{\circ}$ and $15^{\circ}$ to their phase components for short periods of time. The PSE- $\Phi$ algorithm was used, which corrected the biases. Note that in Fig. 6, $\theta_{1}$ is plotted without the angle bias. The result of the bias estimation is shown in Fig. 7, which shows the efficacy of the PSE- $\Phi$ algorithm. The residual error of the least-squares estimation is also shown in Fig. 7. Fig. 8 shows the estimated currents and the measured data, and Fig. 9 shows the absolute error between the measurements and the estimates.

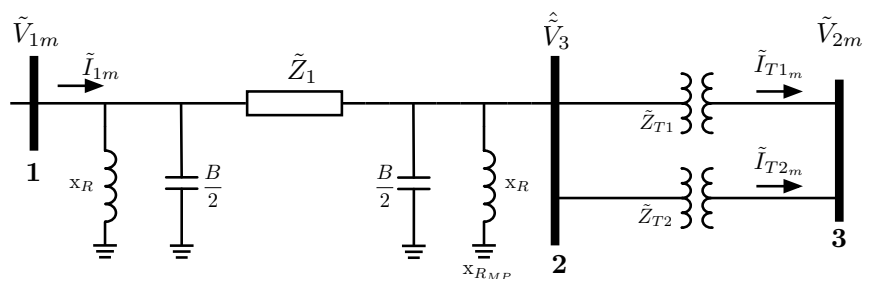

Figure 5. Transmission Path One-Line Diagram

\section{Conclusions}

In this paper, a new approach to state estimation using synchronized voltage and current phasor data is proposed. An algorithm to construct observable phasor state estimator islands is developed. A least-squares approach using synchronized phasor data is formulated, using phasor magnitude and angle variables as the unknowns. The formulation readily extends to the detection and correction of angle biases that may exist in the measured data. Observability analysis using the rank of the Jacobian matrix is proposed and illustrated with several small example networks. The PSE- $\Phi$ method is demonstrated using measured phasor data from the New York power system, and is able to correct the angle biases artificially added to the measured data.

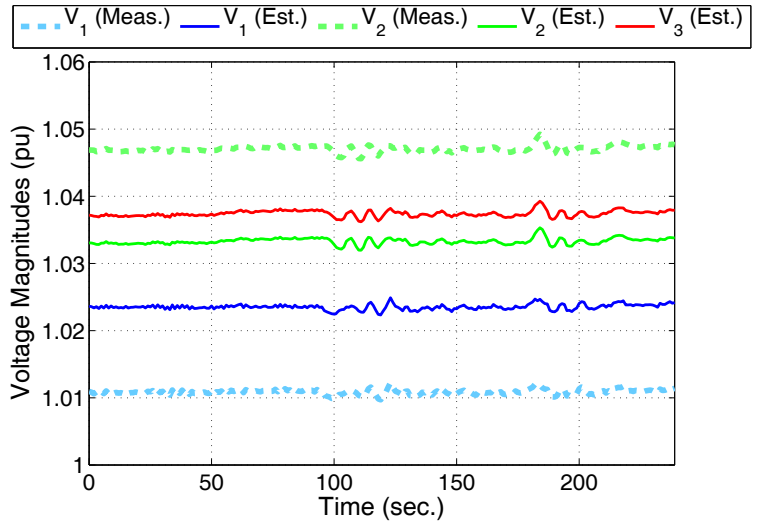

(a) Voltage Magnitudes $\left(V_{i}\right)$

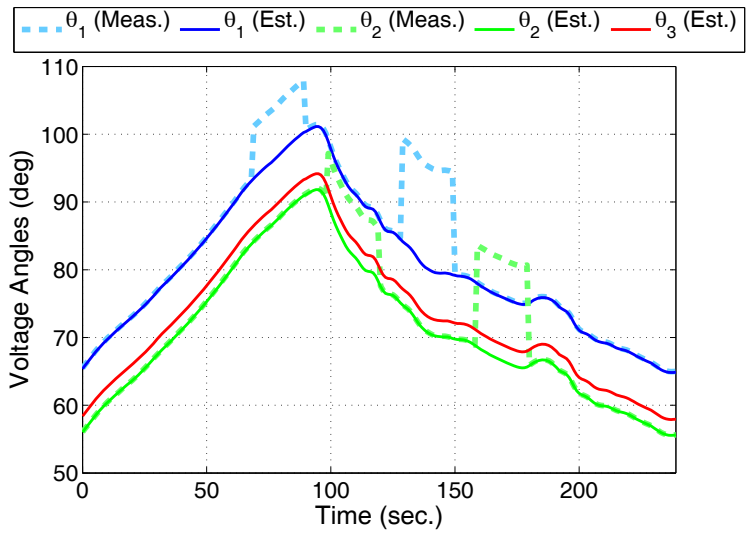

(b) Voltage Angles $\left(\theta_{i}\right)$

Figure 6. Measured and Estimated Voltages

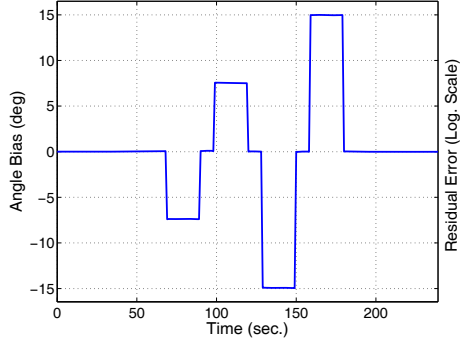

(a) Angle Bias $(\phi)$

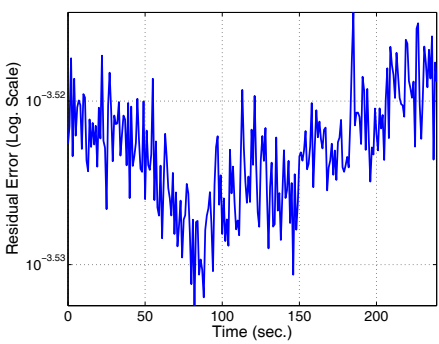

(b) Residual Error
Figure 7. Angle Bias $(\phi)$ and Residual Error under PMU Data with Bias

\section{ACKNOWLEDGMENT}

This work is supported in part by the RPI Power System Research Consortium Industry Members: AEP, FirstEnergy, NE-ISO, NYISO, and PJM, and by NSF through grant ECS0622119. 


\section{REFERENCES}
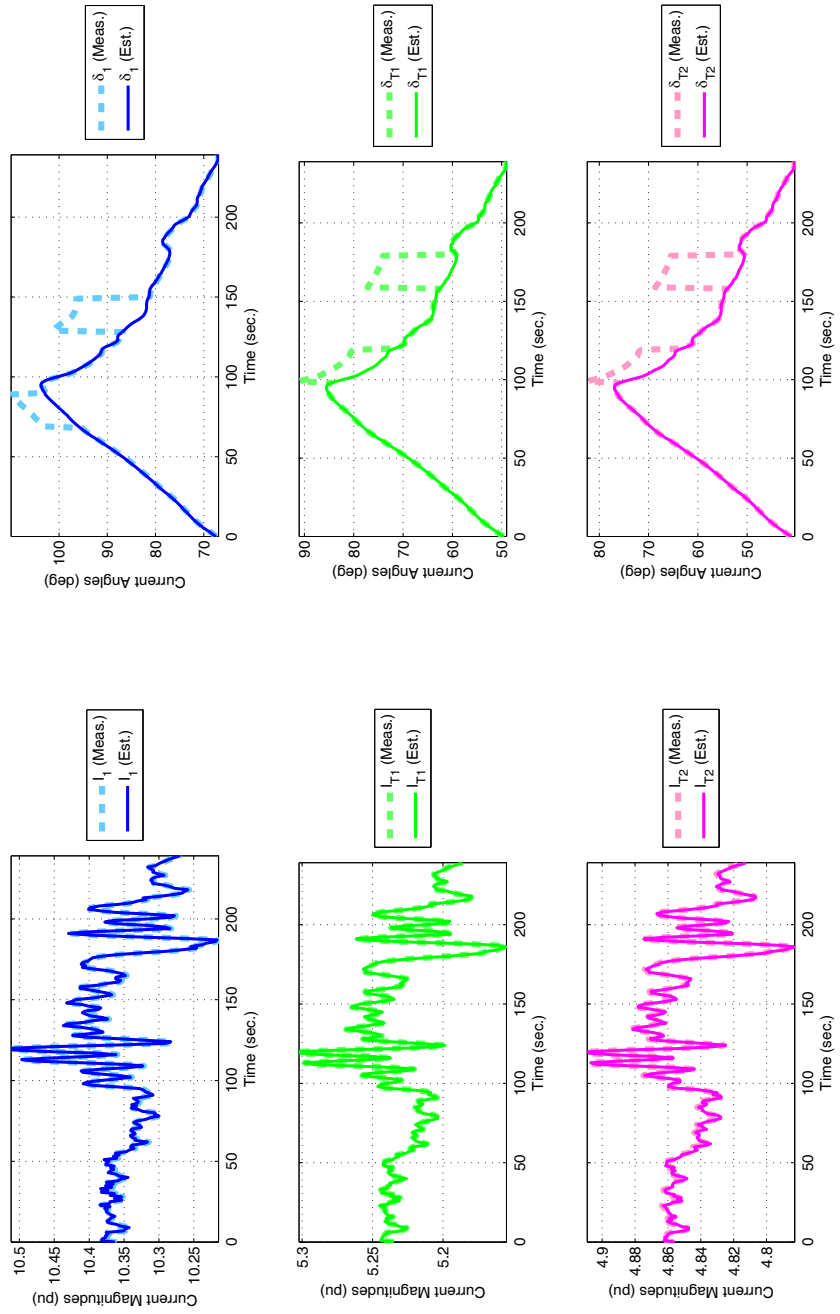

Figure 8. Measured and Estimated Currents
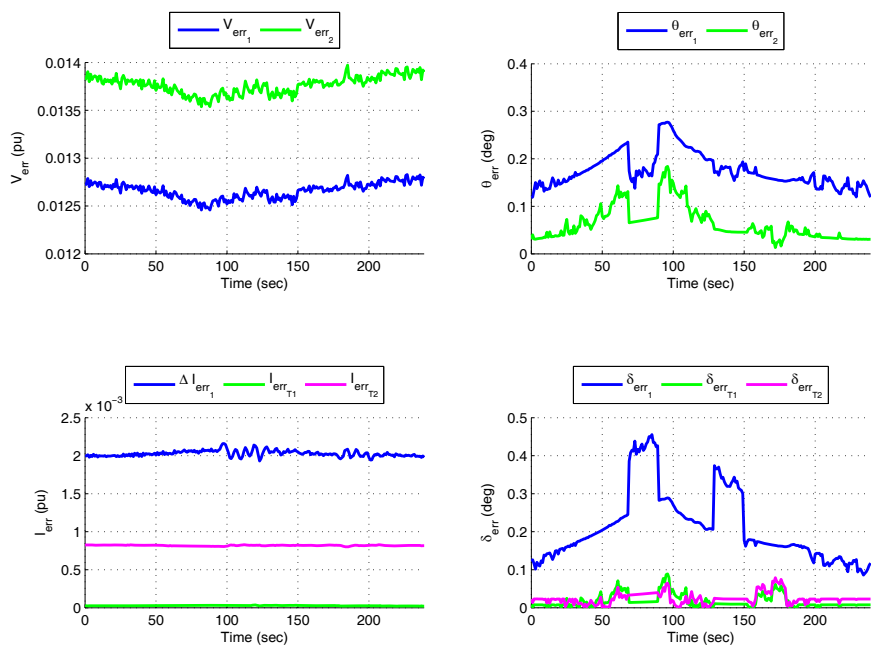

Figure 9. Absolute Error between Measured and Estimated States
[1] F. Schweppe and E. Handschin, "Static State Estimation in Electric Power Systems," Proceedings of the IEEE, vol. 62, no. 7, pp. 972-982, 1974.

[2] A. Monticelli, State Estimation in Electric Power Systems: A Generalized Approach. Springer, 1999.

[3] A. Abur and A. G. Expósito, Power System State Estimation: Theory and Implementation. CRC Press, 2004.

[4] J. Allemong, L. Radu, and A. Sasson, "A fast and reliable state estimation algorithm for aep's new control center," IEEE Transactions on Power Apparatus and Systems, vol. PAS-101, no. 4, pp. 933-944, April 1982.

[5] H. Merrill and F. Schweppe, "Bad Data Suppression in Power System Static Estimation," IEEE Transactions on Power Apparatus and Systems, no. 6, pp. 2718-2725, Nov. 1971.

[6] A. Phadke and J. Thorp, Synchronized Phasor Measurements and Their Applications. New York: Springer, 2008.

[7] A. G. Phadke, J. S. Thorp, and K. J. Karimi, "State estimlatjon with phasor measurements," Power Systems, IEEE Transactions on, vol. 1, no. 1, pp. 233-238, Feb. 1986.

[8] J. Chen and A. Abur, "Placement of pmus to enable bad data detection in state estimation," Power Systems, IEEE Transactions on, vol. 21, no. 4, pp. 1608-1615, Nov. 2006.

[9] L. Vanfretti, J. H. Chow, S. Sarawgi, and D. Ellis, "Phasor state estimation," in North American SynchroPhasor Initiative (NASPI) Work Group Meeting, Oct. 16-17, 2008, Charlotte, NC, available online: http://www.naspi.org/meetings/workgroup/workgroup.stm.

[10] A. Bjorck, Numerical Methods for Least Squares Problems. Philadelphia, PA: SIAM, 1996.

[11] S. Nash and A. Sofer, Linear and Nonlinear Programming. New York: McGraw-Hill, 1996.

Luigi Vanfretti Luigi Vanfretti (S'03) received the Electrical Engineering degree from Universidad de San Carlos de Guatemala in 2005. He is pursuing the Ph.D. degree in the Electrical, Computer, and Systems Engineering Department at Rensselaer Polytechnic Institute, from where he obtained his MS in 2007. He was a visiting researcher at the Department of Electronics and Electrical Engineering of The University of Glasgow, Scotland, in Fall 2005. His research interests are modeling, dynamics, and stability of power systems; applications of PMU data and open source software for power system analysis.

Joe H. Chow (F'92) received his MS and $\mathrm{PhD}$ degrees from the University of Illinois, Urbana-Champaign. After working in the General Electric Power System business in Schenectady, he joined Rensselaer Polytechnic Institute in 1987. He is a professor of Electrical, Computer, and Systems Engineering and the Associate Dean of Engineering for Research and Graduate Programs. His research interests include multivariable control, power system dynamics and control, voltage-sourced converter-based FACTS Controllers, and synchronized phasor data.

Sanjoy Sarawgi (M'03) received his B. Tech (Hons) from Indian Institute of Technology, Kharagpur, India, in 2002 and MS from Washington State University in 2004.Since 2004, he is with the Advanced Transmission Studies and Technologies section of American Electric Power in Columbus, OH. Mr. Sarawgi is a member of the IEEE Power Engineering Society (PES).

Dean Ellis is manager of Short Term Planning for the New York Independent System Operator, Inc, located in Rensselaer, NY. He received his B.S. in Electrical Power Engineering from Rensselaer Polytechnic Institute in 1991. Mr. Ellis is a registered Professional Engineer in New York.

Behruz (Bruce) Fardanesh received his B.S. in Electrical Engineering from Sharif University of Technology in Tehran, Iran in 1979. He also received his M.S. and Doctor of Engineering degree both in Electrical Engineering from the University of Missouri-Rolla and Cleveland State University in 1981 and 1985, respectively. Since 1985 he has been teaching at Manhattan College where he holds the rank of Associate Professor of Electrical Engineering. Currently, he is also working in the area of Advanced Power Delivery in Research and Technology Development at the New York Power Authority. His areas of interest are power systems dynamics, control and operation. 